\title{
PIXEL VS OBJECT-BASED IMAGE CLASSIFICATION TECHNIQUES FOR LIDAR INTENSITY DATA
}

\author{
Nagwa El-Ashmawy ${ }^{\text {ab }}$, Ahmed Shaker ${ }^{\text {a, *, Wai Yeung Yan }}{ }^{\text {a a }}$ Ryerson University, Civil Engineering Department, Toronto, Canada - \\ (nagwa.elashmawy, ahmed.shaker, waiyeung.yan)@ ryerson.ca \\ ${ }^{\mathrm{b}}$ Survey Research Institute, National Water Research Center, Cairo, Egypt
}

\section{Commission VI, WG VI/4}

KEY WORDS: LiDAR, intensity data, object-based classification, decision tree.

\begin{abstract}
:
Light Detection and Ranging (LiDAR) systems are remote sensing techniques used mainly for terrain surface modelling. LiDAR sensors record the distance between the sensor and the targets (range data) with a capability to record the strength of the backscatter energy reflected from the targets (intensity data). The LiDAR sensors use the near-infrared spectrum range which provides high separability in the reflected energy by the target. This phenomenon is investigated to use the LiDAR intensity data for land-cover classification. The goal of this paper is to investigate and evaluates the use of different image classification techniques applied on LiDAR intensity data for land cover classification. The two techniques proposed are: a) Maximum likelihood classifier used as pixelbased classification technique; and b) Image segmentation used as object-based classification technique. A study area covers an urban district in Burnaby, British Colombia, Canada, is selected to test the different classification techniques for extracting four feature classes: buildings, roads and parking areas, trees, and low vegetation (grass) areas, from the LiDAR intensity data. Generally, the results show that LiDAR intensity data can be used for land cover classification. An overall accuracy of $63.5 \%$ can be achieved using the pixel-based classification technique. The overall accuracy of the results is improved to $68 \%$ using the objectbased classification technique. Further research is underway to investigate different criteria for segmentation process and to refine the design of the object-based classification algorithm.
\end{abstract}

\section{INTRODUCTION}

Currently Light Detection and Ranging (LiDAR) becomes one of the most important remote sensing techniques for 3D data acquisition of the Earth surface, (Song et al, 2002, Brennan and Webster, 2006, Hui et al., 2008, and Yan \& Shaker, 2010). LiDAR acquires highly precise and accurate vertical and horizontal data $( \pm 30 \mathrm{~cm})$, (Brennan and Webster, 2006). This high accurate data are used for generating digital elevation and/or surface models (DTM/DSM). LiDAR data have been used intensively in the 3D City modelling and building extraction and recognition (Haala \& Brenner, 1999, Song et al, 2002, and Yan \& Shaker, 2010). The concept of LiDAR sensors is based on sending laser pulses toward objects and receiving the reflected signals. The distances between the targets and the laser sensor (range data) and the 3D coordinates (x, y, and $\mathrm{z}$ ) for points cloud are, consequently, calculated with the aid of other sensors (GPS, and IMU), (Ackreman, 1999).

Traditionally, classification of LiDAR data referred to the separation of terrain point from other objects (non-terrain point) based on the elevations, (Antonarakis et al., 2008). Kraus \& Pfeifer, (1998) have used LiDAR data to create DTM in wooded areas. The accuracy of the DTM extracted was $25 \mathrm{~cm}$ for flat areas, which is improved to $10 \mathrm{~cm}$ by refining the data processing method. The work includes automatic classification of the laser point cloud into terrain and vegetation points using special filtering and interpolation techniques. With the capability to record the intensity of the reflected energy, definition of the classification of LiDAR data includes the use of the intensity data as well as the range in the LiDAR classification. Haala \& Brenner (1999) combined LiDAR elevation data and a multi-spectral aerial photo (Green, Red and NIR bands) for building extraction using unsupervised classification technique. It was found that combining the multispectral aerial photo with the LiDAR elevation data improved the classification results significantly.

LiDAR sensors use Near Infrared (NIR) spectrum range to record the backscatter energy reflected from the targets (intensity data). A NIR image can be generated by interpolating the intensity data collected by the LiDAR sensor. Intensity data is investigated to be used to distinguish different target materials using various image classification techniques. In the last decade, researchers studied the integration of the LiDAR intensity and range data for data classification and feature extraction. The intensity data were used primarily as complementary data instead of the traditional multi-spectral remote sensing imageries (such as aerial photos and satellite images) for data visualization and interpretation. LiDAR intensity data are advantageous over the multi-spectral remote sensing data in avoiding the shadows appear in the multispectral data that is because LiDAR sensor is an active sensor. Hui et al., 2008, used the intensity and height LiDAR data for

\footnotetext{
* Corresponding author:

Ahmed Shaker, Ryerson University, Department of Civil Engineering,

350 Victoria St., Toronto, Canada, M5B 2K3

E-mail: ahmed.shaker@ryerson.ca

Tel: +1 4169795000 (ext. 4658)
} 
land-cover classification. Supervised classification technique was used to differentiate four classes: Tree, Building, Bare Earth and Low Vegetation. It was observed that combining the intensity data with the height data is an effective method for LiDAR data classification. However, quantitative accuracy assessment was not included in that research work.

Recent researchers combine the laser data with other auxiliary data such as multispectral aerial photos or satellite images, USGS DEM, texture data, normalized height, and multiplereturns data. Charaniya et al, (2004) used LiDAR height and intensity data, height variation data, multiple-return data, USGS DEM, and luminance data of a panchromatic aerial imagery for land-cover classification. A supervised classifier was used to distinguish four classes: trees, grass, roads, and roofs. The effect of band combinations on the classification results was studied. It was observed that height variation affected positively the classification results of the high vegetation areas, Luminance and intensity data was useful for distinguishing the roads from the low vegetation areas, and the multiple-return differences slightly improved the classification of roads and buildings but reduced the accuracy of the other classes.

A rule-based approach was presented by Bartels, (2006) to improve the accuracy of the classification obtained by supervised Maximum Likelihood classification for the LiDAR data (first and last echo and intensity data) that are fused with other co-registered bands (aerial and near Infrared bands). Four classes were identified: Buildings, Vegetation, Cars and Ground. The accuracy obtained by this approach varied based on the bands used in the classification. The overall accuracy of the classification of the intensity data, and height data were 36 and $51 \%$, respectively. Combining the two data sets improve the overall accuracy to $62 \%$. The accuracy obtained reaches $71 \%$ when the first and last echo data are used. Using the coregistered data improve the accuracy by $13-33 \%$, (Bartels, 2006).

Subsequently, researchers have paid more attention to the intensity data and started to analyse the data and study different enhancing methods to remove the noise and improve the data interpretation. Song et al, (2002) examined different resampling techniques to convert LiDAR point data to grid image data which is filtered to remove the noise with minimum influence on the original data. The resampled grid is used to investigate the applicability of using the LiDAR intensity data for landcover classification. It is conclude that the LiDAR intensity data contain noise that is needed to be removed.

Radiometric correction of the intensity data was suggested in some of the recent literatures (Coren and Sterzia, 2006; Höfle and Pfeifer, 2007). The process mainly relies on the use of the laser range equation to convert the intensity data into the spectral reflectance with consideration of the scanning geometry, the atmospheric attenuation, and the background backscattering effects. After the radiometric correction, the homogeneity of the land cover is improved and thus enhances the performance of feature extraction and surface classification. Yan et al. (2011) evaluated the accuracy of different land cover classification scenarios by using the airborne LiDAR intensity data before and after radiometric correction. An accuracy improvement of $8 \%$ to $12 \%$ was found after applying the radiometric correction.

The previous studies conducted the land-cover classification using the common parametric classifiers, such as the Maximum
Likelihood. Nevertheless, these parametric classifiers are considered as pixel-based classification, which might not be efficient with high resolution images, according to Blaschke, (2010). Object-based classification using image segmentation is proposed for land cover classification of the high spatial resolution imageries. LiDAR intensity images can be considered as high spatial resolution images. The object-based approach for LiDAR intensity data classification has been investigated in several studies. In Brennan and Webster, (2006), the objectbased classification is used for distinguishing ten classes of land cover, including saturated and non-saturated intertidal sediments, saturated or stressed and lush ground cover vegetation, low and tall deciduous and coniferous trees, roads and bare soil, bright-roofed structures, dark-roofed structures, and water. The classification approach was applied on five different images generated from the LiDAR data, DSM, DTM, Normalised height, intensity, and multiple return data. The overall accuracy of the ten classes is $94 \%$ increased to $98 \%$ when the classes aggregated to seven classes, (Brennan and Webster, 2006).

Antonarakis et al., (2008) followed different object-oriented approach for land cover classification. This classification used point distribution frequency criteria such as skewness and kurtosis to differentiate between the land cover classes. This study verified that a combination of intensity and elevation LiDAR data can be used for multiple land-cover classification. It showed that high accuracy of $95 \%$ can be achieved for forest areas.

This research investigates the use of the intensity data for landcover classification. Different classification techniques are proposed, and classification accuracy is assessed to further improve the results. The paper divided into five sections. Section 1 is the introduction and highlights on the previous work. Section 2 comprises the methodology used in this research. Section 3 is a description to the study area and the data sets used. Section 4 includes the results of the experimental work and further analysis. The paper is concluded by a summary of the work and the future work.

\section{METHODOLOGY}

The possibility of using the LiDAR intensity data in land cover classification is investigated. Two classification approaches are examined and their results are compared for further assessment. The two approaches are pixel-based and object-based classification approaches. First, the pixel-based classification approach presented by the maximum likelihood classification technique is used to classify the LiDAR intensity data into number of information classes. Then, more bands such as DSM, texture of the intensity data, and terrain slope are added, as different bands, to the intensity data to examine any improvement in the classification accuracy. Second, image segmentation is introduced for the object-based classification approach. A decision tree with certain criteria is developed to distinguish the different information classes. These criteria are based on the segmentation of the intensity data taking into consideration the surface elevation data. The results of the two approaches are evaluated and assessed for future improvement. Details of the two classification approaches are discussed in the following sections. 


\subsection{Pixel-Based Classification}

Maximum Likelihood classifier is selected as a pixel-based supervised classification algorithm that considers the probability and class variability of the classes, (Jensen, 2005).

The procedure of the Maximum Likelihood pixel-based classifier is: First, intensity data is imported as a single band image. Second, training signatures are identified for the four different classes. Statistical assessments of the training signatures are done and further enhancement to the selection of the training areas are taken place. Then, the Maximum Likelihood algorithm is applied and the intensity data is classified into the corresponding classes. In similar way, additional bands (DSM, texture, and slope) are imported and included in the process and the results are evaluated and assessed for the production of the Land Cover classes. The classification process is evaluated using more than 1000 reference points that are randomly selected from the original point cloud data to avoid the effect of the interpolation on the accuracy of the ground truth. These points are well-distributed over the study area. The ground truth information is collected from the ortho-rectefied aerial photo provided with the LiDAR data. The Erdas Imagine version 10 is used to conduct the pixelbased classification.

\subsection{Object-Based Classification}

The LiDAR intensity data has interpolated to an intensity image of $20 \mathrm{~cm}$ spatial resolution. This intensity image is considered as a high resolution image; accordingly, the pixel-based classification may not be the best classification technique for such high spatial resolution data, (Blaschke, 2010). Therefore, the object-based classification approach, presented by image segmentation technique, is followed. Segments are homogeneous regions generated based on one or more criteria of homogeneity. Segmentation, also, relates each pixel to the surrounding neighbours, which gives it additional spatial advantages over the single pixel, (Blaschke, 2010).

Similar to the sequence of the work with pixel-based classification, the intensity data are used primarily and then additional bands such as DSM are used in the image classification process. The object-based classification is executed in two levels. First, the intensity image is segmented into homogeneous and heterogeneous areas in order to gather all neighbour pixels in one area. To define the variation in brightness values to be used for segmentation criterion, several trials have been done. The results of these trials are compared and the best difference in the brightness values that separates the objects is 3 . The split and merge segmentation method is used. Area size equals to $100 \mathrm{~m}^{2}$ is used as a criterion to separate the homogeneous surfaces from the heterogeneous ones. This criterion, defining the minimum homogeneous area, is established based on the common minimum size of buildings in the urban areas in Canada. Moreover, the DSM is separated into Terrain and Objects (non-terrain) based on the elevation values, (the elevation value criterion will be different for different study area). As a result from this step, four classes are defined, man-made features, represented by the homogeneous areas, vegetation features, represented by heterogeneous areas, terrain (low elevation areas), and objects (high elevation areas), as shown in Figure 1, Level 1. Secondly, by intersecting each of the homogeneous and heterogeneous super-classes with each of the Objects and Terrain super-classes, the four final classes are formed as shown in Figure 1, Level 2. Two man-made classes, buildings and roads (homogeneous areas with high and low elevations), and two vegetation classes; trees and grass (heterogeneous areas with high and low elevations).

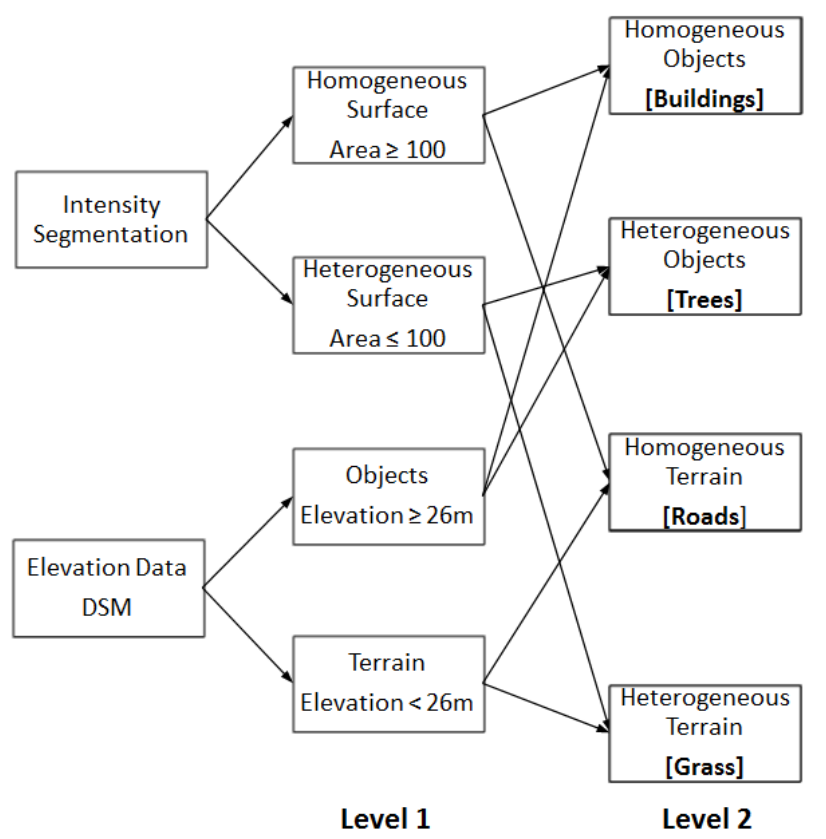

Figure 1: Object-Based Classification Decision Tree

\section{STUDY AREA AND DATA SETS}

\subsection{Study Area}

The study area covers the British Columbia Institute of Technology (BCIT), which is located in the Burnaby, British Columbia, Canada $\left(122^{\circ} 59^{\prime} \mathrm{W}, 49^{\circ} 15^{\prime} \mathrm{N}\right)$. An area of $500 \mathrm{~m} \mathrm{x}$ $400 \mathrm{~m}$ is clipped for the experimental work (Figure 2). This area is selected because it contains a variety of the land cover features on the ground including; buildings, parking areas, trees and open spaces with grassy coverage. The West side of the study area is covered by trees and is higher in elevation comparing to the South and the North parts of the study area by around $10 \mathrm{~m}$.

\subsection{Data Sets}

A Leica ALS50 sensor operating in $1.064 \mu \mathrm{m}$ wavelength and 0.33 mrad beam divergence is used to acquire the LiDAR data. The LiDAR acquisition mission was conducted on July 17, 2009 at local time 14:55. The data acquired contains a 3D point cloud ( $x, y$, and $z$ coordinates) and linearized intensity values (I) for each point, (Figure 3 ). The study area is covered by around million points of the LiDAR data.

About one million points are extracted from the data sets for this study. The data sets used are: a) geometrically calibrated and radiometrically corrected LiDAR data (including $\mathrm{x}, \mathrm{y}, \mathrm{z}$ and I), b) Digital surface model (DSM) generated from the range measurements and converted into raster data using Kriging interpolation algorithm, and c) an Ortho-rectified aerial photo acquired at the same time of the LiDAR data, all data sets are rasterized with pixel size equals to $20 \mathrm{~cm}$. (Figure 4). 


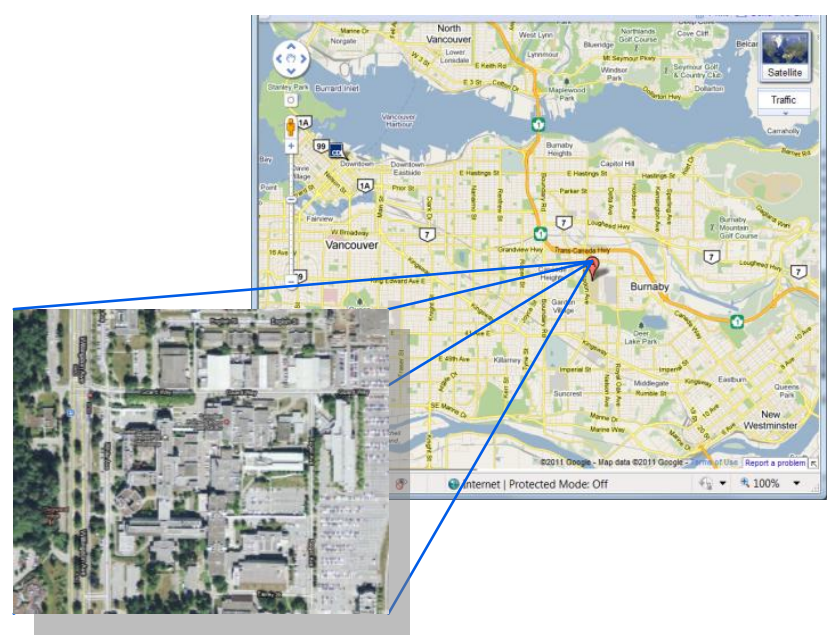

Figure 2: Study Area (British Colombia Institute of Technology, Vancouver)

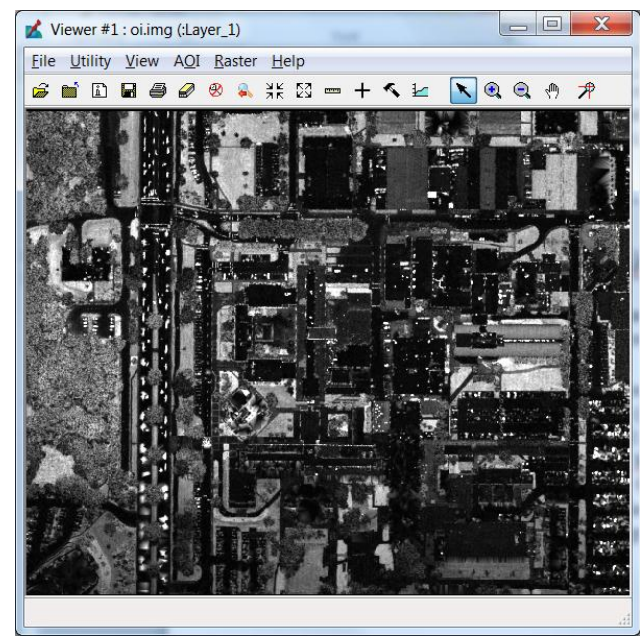

Figure 3: Raster Image of the Original LiDAR Intensity Data

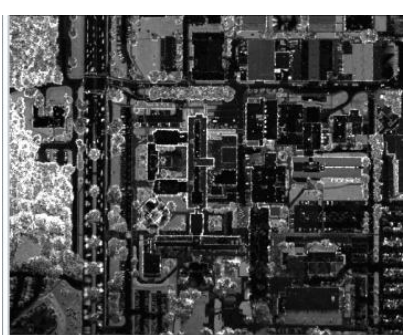

A

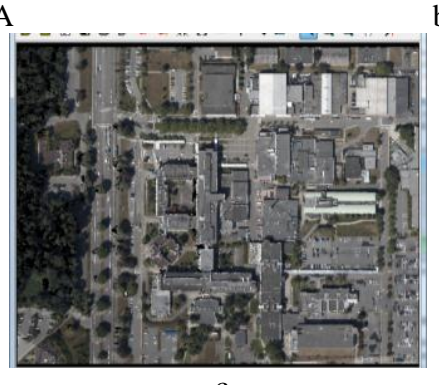

$\mathrm{c}$

Figure 4: Geometrically Calibrated and Radiometrically corrected Intensity Image (a), DSM (b), and Ortho-rectified Aerial Photo (c)

\section{RESULTS AND DISCUSSION}

After examining the study area and the provided data sets, it was noticed that:

- Areas covered by trees and grass have brighter values of the intensity data comparing to areas covered by buildings and roads. This is expected as the used signals are in near Infra-Red range, which has high reflectance from vegetation.

- The area at the West South corner is higher in elevation than the Northern and Eastern areas. This difference in terrain elevation is expected to have negative effect on the results of the classification when the DSM data are included. The reason is that grass in high terrain will be difficult to be distinguished from trees.

- The intensity values of the buildings and roads are more homogeneous than it is in the trees and the grasses.

- The surface of each building has constant slopes for large area. On the other hand, the slope of the tree area is not constant.

\subsection{Results of Pixel-Based Classification}

Based on the previous observations, it can be noted that intensity data can be effectively used for distinguishing manmad objects from the vegetation. Generating different bands and including them in the classification process is expected to improve the classification results. These bands can be summarized as; texture image for the intensity data, slope image of the DSM to represent the roof slopes, and modified DSM in such a way that avoids the effect of the terrain elevation on the classification created by subtracting an inclined surface from the DSM. The inclined surface is generated from few points located on the roads at the four corners of the study area. This modification of the DSM depends on the data set. For more accurate results the multi-return data have to be used to avoid the terrain elevation effects on the data; however these data are not available for this study. Figure 5 illustrates the bands used in the study.

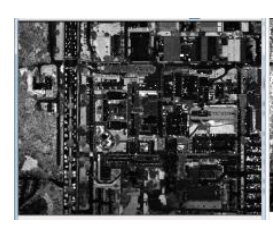

i

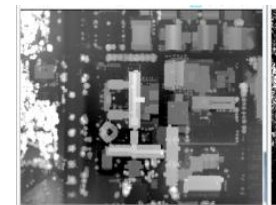

iv

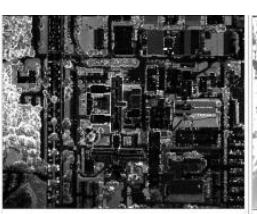

Ii

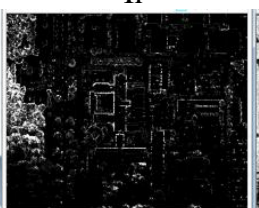

V

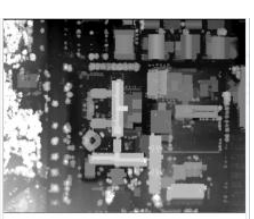

Iii

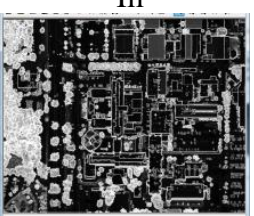

$\mathrm{Vi}$
Figure 5: Bands used in the study i) raw data, ii) Geometric Calibrated and Radiometric corrected Intensity Data, iii) DSM, iv) Modified DSM, v) Intensity Texture, and vi) DSM slope

Five combinations of bands are developed to study the effects of the auxiliary data on the classification results. These combinations are: a) Intensity image only, b) Intensity image and DSM, c) Intensity image and Modified DSM (after eliminating the terrain height effects), d) Intensity image, 
Modified DSM, and Intensity Texture image, and e) Intensity image, Modified DSM, Texture, and DSM slope image.

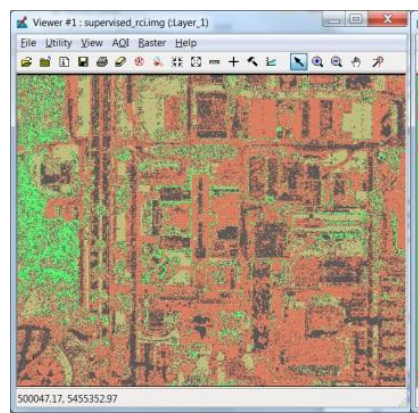

(a)

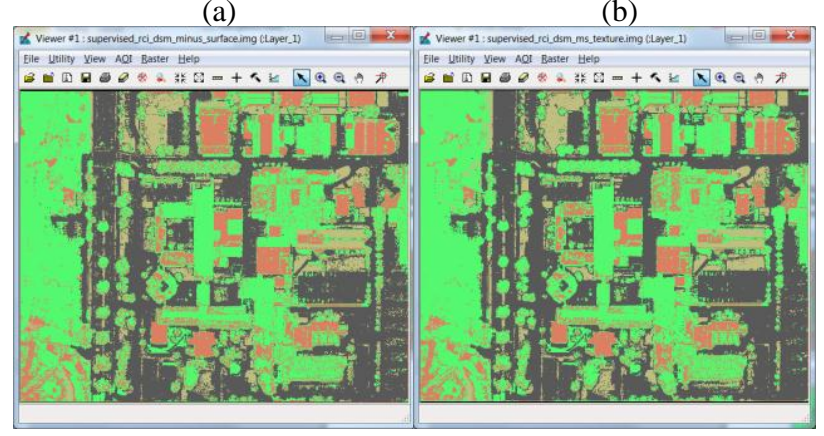

(c)

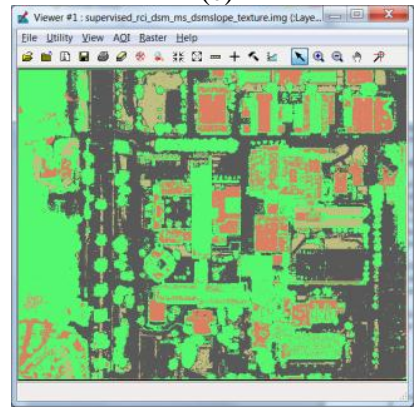

(e)
Figure 6: Results of Pixel-Based Classification

The results of the five combinations are illustrated in Figure 6, the overall accuracy for each case are listed in Table 1, and the individual classes accuracies are represented in Table 2. From the results obtained, the overall accuracy from classifying the intensity data only to two classes is $71 \%$ which is decreased to $43 \%$ considering 4 classes (roads, building, trees, and grass). It is obvious that depending only on the intensity data, man-made features can be distinguished from natural features. However, one cannot distinguish the buildings from the roads in the manmade features or the grass from the trees in the natural features, since both pairs of classes have similar spectral reflectance characteristics in the Near Infra-red spectrum range. As a result, the intensity data can be used to distinguish the man-made features from the vegetation, yet additional information is required to differentiate between the roads and buildings in the man-made features, and between the trees and the grass in the vegetation features. Adding the elevation data, a significant improvement in the overall accuracy of the classification results is achieved (increases from $42.97 \%$ to $52.97 \%$ ), Figures $6 \mathrm{a}$ and $6 \mathrm{~b}$.

Nevertheless, it is clear from Figure 6b that the South Western area is classified incorrectly as trees. That is because this area has higher elevation, which affects the classification results. Therefore, the modified DSM is used instead of the provided one. This improves the overall accuracy of the results from $52.97 \%$ to $59.21 \%$, Figures $6 \mathrm{~b}$ and $6 \mathrm{c}$. Based on the previous observation that the buildings and roads have more homogeneous intensity data than the trees and the grass, the overall accuracy of the classification is increased when the texture of the intensity data are used (from $59.21 \%$ to $64.12 \%$ ), Figures $6 \mathrm{c}$ and $6 \mathrm{~d}$. No further improvements to the accuracy of the classification results are found when the slope of the surfaces is considered in the classification process $(64.12 \%$ and $63.74 \%$ ), Figures $6 \mathrm{~d}$ and $6 \mathrm{e}$. It can be concluded that using the elevation data does improve the classification results significantly. Moreover, other auxiliary data such as texture of intensity data have an essential role in improving the classification results.

Table 1: Accuracy Assessment of Pixel-Based Classification

\begin{tabular}{|c|l|l|}
\hline Case & Band Combination & $\begin{array}{l}\text { Overall } \\
\text { Accuracy }\end{array}$ \\
\hline a & Intensity Band & $42.97 \%$ \\
\hline b & Intensity, DSM Bands & $52.97 \%$ \\
\hline c & Intensity, Modified DSM Bands & $59.21 \%$ \\
\hline d & Intensity M-DSM, Texture Bands & $64.12 \%$ \\
\hline e & Intensity M-DSM, Texture Slope Bands & $63.74 \%$ \\
\hline
\end{tabular}

Table 2: Producer and User Accuracies of the Pixel-Based

\begin{tabular}{|l|c|c|}
\multicolumn{3}{|c}{ Classes } \\
\hline Class Name & Producers Accuracy. & Users Accuracy. \\
\hline Tree & $92.74 \%$ & $59.63 \%$ \\
\hline Build & $40.61 \%$ & $91.18 \%$ \\
\hline Grass/Soil & $46.00 \%$ & $74.68 \%$ \\
\hline Road & $78.33 \%$ & $66.45 \%$ \\
\hline
\end{tabular}

\subsection{Results of Object-Based Classification}

Figure 7 shows the produced land-cover classes from the object-based classification process. It is found that there are several buildings, which were classified as trees in the pixelbased classification, are classified correctly as buildings in the object-based classification. To assess the overall accuracy of the results, the same 1000 reference points that were used in the pixel-based classification assessment are used here. The overall accuracy of the object-based classification is increased to $65.91 \%$. The individual class accuracies are shown in Table 3.

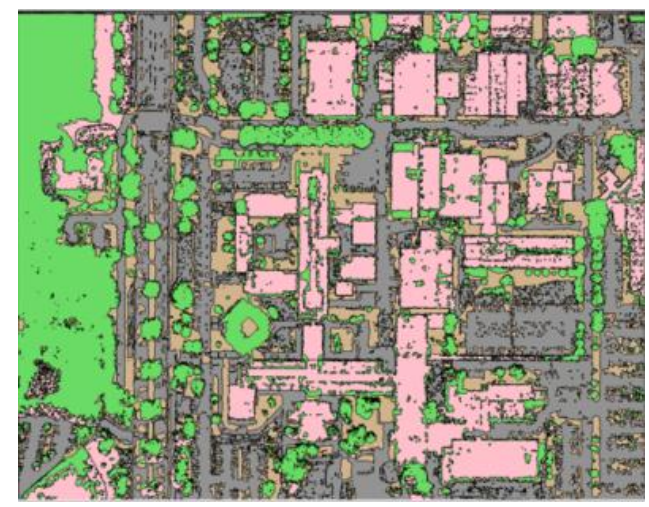

Building

Trees

Roads Grass

Figure 7: Results of Object-Based Classification 
Table 3: Producer and User Accuracies of the Pixel-Based Classes

\begin{tabular}{|l|l|l|}
\hline Class Name & Producers Accuracy. & Users Accuracy. \\
\hline Tree & $88.33 \%$ & $75.47 \%$ \\
\hline Build & $67.25 \%$ & $76.24 \%$ \\
\hline Grass/Soil & $42.80 \%$ & $45.34 \%$ \\
\hline Road & $59.70 \%$ & $62.55 \%$ \\
\hline
\end{tabular}

The accuracy obtained is higher than the one obtained from applying the pixel-based classification; however, it is less than expected. This may be due to the results achieved which classified the buildings as unfilled polygons where some of the check points fall within holes in the classified building polygons giving incorrect results, and the misclassification of the some small roof structures. The results are expected to be improved by using the multi return data for separating the terrain and objects. Further researches are underway to improve the representation of the objects through improving the design of the decision.

\section{CONCLUSIONS}

LiDAR intensity data have been examined to be used for LandCover classification. Both Pixel-based and Object-based classification techniques are used to classify the LiDAR intensity data. From the results obtained, it can be concluded that the intensity data can be used successfully to distinguish the vegetation from the man-made features (overall accuracy $=$ $71 \%$ ). However to distinguish trees from grass and roads from buildings, auxiliary data are required. Adding auxiliary data, such as DSM, Texture of the intensity data and slope of surfaces improves the accuracy of the pixel-based classification from $43 \%$ to $64 \%$. Classifying the LiDAR intensity data using the object-based classification further improves the accuracy of the classification results. A special decision tree is designed for this research work considering the intensity and the elevation data. The classification accuracy using the object-based classification approach reached $66 \%$. Further research work is underway to further improve the classification accuracy.

\section{ACKNOWLEDGMENT}

This research work is supported by the Discovery Grant from the Natural Sciences and Engineering Research Council of Canada (NSERC) and the GEOIDE Canadian Network of Excellence, Strategic Investment Initiative (SII) project SII PIV \# 72. The authors would like to thank McElhanney Consulting Services Ltd, BC, Canada for providing the real LiDAR and image datasets

\section{REFERENCES}

1. Ackerman, F., 1999. Airborne Laser Scanning-Present Status and Future Expectations. ISPRS Journal of Photogrammetry \& Remote Sensing, 54, No. 2-3, pp. 6467.

2. Antonarakis, A., Richards, K., Brasington, J., 2008. Object-Based Land-Cover Classification Using Airborne LiDAR. Remote Sensing of Environment 112 (2008) 2988-2998.

3. Bartels, M., Wei, H., and Ferryman, J. 2006. Analysis of LiDAR Data Fused with Co-Registered Bands. In
Proceedings of the IEEE International Conference on Video and Signal Based Surveillance, (AVSS'06).

4. Beasy, C., Hopkinson, C., and Webster, T., 2005. Classification of Nearshore Materials on the Bay of Fundy Coast Using LiDAR Intensity Data. In Proceedings of the Canadian Symposium for Remote Sensing, Wolfville, June 2005.

5. Blaschke, T., 2010. Object Based Image Analysis for Remote Sensing. ISPRS Journal of Photogrammetry \& Remote Sensing, 65 (21010) 2-16.

6. Brennan, R., and Webster, T.L., 2006. Object-Oriented Land Cover Classification of LiDAR-derived Surfaces. Can. J. Remote Sensing, Vol. 32, No. 2, pp. 162-172.

7. Charaniya, A., Manduchi, R., and Lodha, S., 2004. Supervised Parametric Classification of Aerial LiDAR Data. In: Proceedings of the 2004 IEEE Computer Society Conference on Computer Vision and Pattern Recognition Workshops (CVPRW'04).

8. Coren, F., and Sterzai, P., 2005. Radiometric Correction in Laser Scanning. International Journal of Remote Sensing, 27(15), pp. $3097-3014$.

9. Haala, N., Brenner, C., 1999. Extraction of Buildings and Trees in Urban Environments. ISPRS Journal of Photogrammetry \& Remote Sensing, 54 (1999) 130-137.

10. Höfle, B., and Pfeifer, N., 2007. Correction of laser scanning intensity data: Data and model-driven approaches. ISPRS Journal of Photogrammetry \& Remote Sensing, 62(6): 415-433.

11. Hui, L., Di, L., Xianfeng, H., and Deren, L., 2008. Laser Intensity Used In Classification of LiDAR Point Cloud Data. Geoscience and Remote Sensing Symposium, Boston, Massachusetts, U.S.A.

12. Jensen, J., 2005. Introductory Digital Image Processing, 3/e, Prentice Hall, Upper Saddle River, New Jersey, ISBN 0-13-1453361-0: 526p.

13. Kraus, K., and Pfeifer, N., 1998. Determination of Terrain Models in Wooded Areas with Airborne Laser Scanner Data. ISPRS Journal of Photogrammetry \& Remote Sensing, 53 (1998) 193-203.

14. Song, J., Han, S., Yu, K., and Kim, Y., 2002. Assessing the Possibility of Land-Cover Classification Using LiDAR Intensity Data. ISPRS Commission III, Symposium, Graz, Austria. P.B-259ff.

15. Yan, W., and Shaker, A., 2010. Radiometric Calibration of Airborne LiDAR Intensity Data for Land Cover Classification. Canadian Geomatics Conference and Symposium of Commission I, ISPRS. Calgary, Alberta, Canada.

16. Yan, W.Y., Shaker, A., Habib, A., and Kersting, A. P. 2011. Improving Classification Accuracy of Airborne LiDAR Intensity Data by Geometric Calibration and Radiometric Correction. ISPRS Journal of Photogrammetry and Remote Sensing (Submitted). 